\title{
PT2c Stage Finding
}

National Cancer Institute

\section{Source}

National Cancer Institute. pT2c Stage Finding. NCI Thesaurus. Code C48767.

A pathologic primary tumor TNM stage finding. The definition of pT 2c stage finding depends on the particular type of cancer that it refers to; for example, for prostate cancer, pT 2c stage finding is defined as follows: cancer involving both lobes of the prostate gland; for fallopian tube cancer, pT 2c stage finding is defined as follows: cancer with pelvic extension and malignant cells in ascites or peritoneal washings. (from AJCC 7th Ed.) 